\title{
Health and Environmental Risk Assessment Project for bottlenose dolphins Tursiops truncatus from the southeastern USA. I. Infectious diseases
}

\author{
Gregory D. Bossart ${ }^{1,2, *}$, Patricia Fair ${ }^{3,4}$, Adam M. Schaefer ${ }^{5}$, John S. Reif ${ }^{6}$ \\ ${ }^{1}$ Georgia Aquarium, 225 Baker Street, NW, Atlanta, Georgia 30313, USA \\ ${ }^{2}$ Division of Comparative Pathology, Miller School of Medicine, University of Miami, PO Box 016960 (R-46), Miami, \\ Florida 33101, USA \\ ${ }^{3}$ National Oceanic and Atmospheric Administration, National Ocean Service, Center for Coastal Environmental Health \\ and Biomolecular Research, 219 Fort Johnson Rd, Charleston, South Carolina 29412, USA \\ ${ }^{4}$ Department of Public Health Sciences, Medical University of South Carolina, Charleston, South Carolina 29425, USA \\ ${ }^{5}$ Harbor Branch Oceanographic Institute at Florida Atlantic University, 5600 U.S. 1 North, Fort Pierce, Florida 34946, USA \\ ${ }^{6}$ Department of Environmental and Radiological Health Sciences, College of Veterinary Medicine and Biomedical Sciences, \\ Colorado State University, Fort Collins, Colorado 80523, USA
}

\begin{abstract}
From 2003 to 2015, 360 free-ranging Atlantic bottlenose dolphins Tursiops truncatus inhabiting the Indian River Lagoon (IRL, $\mathrm{n}=246)$, Florida, and coastal waters of Charleston (CHS, $\mathrm{n}=114$ ), South Carolina, USA, were captured, given comprehensive health examinations, and released as part of a multidisciplinary and multi-institutional study of individual and population health. The aim of this review is to summarize the substantial health data generated by this study and to examine morbidity between capture sites and over time. The IRL and CHS dolphin populations are affected by complex infectious and neoplastic diseases often associated with immunologic disturbances. We found evidence of infection with cetacean morbillivirus, dolphin papilloma and herpes viruses, Chlamydiaceae, a novel uncultivated strain of Paracoccidioides brasiliensis (recently identified as the causal agent of dolphin lobomycosis/lacaziasis), and other pathogens. This is the first long-term study documenting the various types, progression, seroprevalence, and pathologic interrelationships of infectious diseases in dolphins from the southeastern USA. Additionally, the study has demonstrated that the bottlenose dolphin is a valuable sentinel animal that may reflect environmental health concerns and parallel emerging public health issues.
\end{abstract}

KEY WORDS: Cetacean · Bottlenose dolphin $\cdot$ Cetacean Morbillivirus $\cdot$ Papilloma $\cdot$ Lobomycosis · Paracoccidioidomycosis ceti $\cdot$ Chlamydiaceae $\cdot$ Arbovirus

\section{INTRODUCTION}

The Atlantic Bottlenose Dolphin Health and Environmental Risk Assessment (HERA) Project was started in 2003 as a multidisciplinary and multi-institutional cooperative effort designed to evaluate individual and population health of bottlenose dolphins

\footnotetext{
*Corresponding author: gbossart@georgiaaquarium.org
}

Tursiops truncatus in 2 southeast US coastal regions: Charleston, South Carolina (CHS), and the Indian River Lagoon, Florida (IRL) (Bossart et al. 2006). Prior to 2003, both geographic areas had evidence of bottlenose dolphin stock depletion, unusual mortality events, epizootic disease, and/or anthropogenic habitat degradation. HERA Project goals were to estab-

() The authors 2017. Open Access under Creative Commons by Attribution Licence. Use, distribution and reproduction are unrestricted. Authors and original publication must be credited. 
lish standardized methodology for measuring dolphin health and risk assessment, to investigate associations between health status and environmental stressors and, if indicated, to use the data to promote environmental improvement and conservation. As a typically long-lived apex predator which often exhibits a relatively high degree of site fidelity, bottlenose dolphins are emerging as an important sentinel species for monitoring environmental health and for evaluating the potential relationship between exposure to infectious agents and anthropogenic contaminants (Bossart 2011). In this paper, we review the health assessment and infectious disease data for the IRL and CHS dolphins from 2003 to 2015. This integrated summary indicates that both dolphin populations have unique health challenges that can impact individual and population health, and it also provides important new information on regional environmental and public health issues.

\section{DOLPHIN STUDY SITE LOCATIONS}

The detailed environmental characteristics of the IRL and CHS study sites are provided elsewhere (Fair et al. 2006a, Reif et al. 2017 [this issue]). Briefly, the IRL is a shallow-water estuary that comprises $40 \%$ of Florida's central east coast (Bossart et al. 2003). The lagoon is an aggregate of 3 estuarine water bodies (Indian River, Banana River, and Mosquito Lagoon) and extends $250 \mathrm{~km}$ from Ponce De Leon Inlet in the north to Jupiter Inlet in the south (Fig. 1).

Previous studies of health in IRL dolphins were based largely on data from stranded animals (Hersh et al. 1990, Stolen 1998). Since the 1970 s, up to $40 \%$ of dolphin strandings along the east coast of Florida have occurred in the IRL (Stolen et al. 2007). Pathologic findings in stranded dolphins from the IRL suggested that a substantial portion of the observed mortality was due to infectious diseases and that immunologic dysfunction was a likely component in disease pathogenesis (Bossart et al. 2003). Importantly, the IRL experienced 3 Unusual Mortality Events (UMEs) (i.e. strandings which occur at significantly elevated rates) in 2001, 2008, and 2013 (Marine Mammal Commission 2002, NOAA Fisheries 2015), for which the cause remained undetermined after investigation. Additionally, IRL dolphins were involved in the 2013-2015 bottlenose dolphin UME in the Mid-Atlantic caused by cetacean morbillivirus (CeMV; see below). The HERA Project was partially designed to provide long-term surveillance for health parameters in the event of a UME.

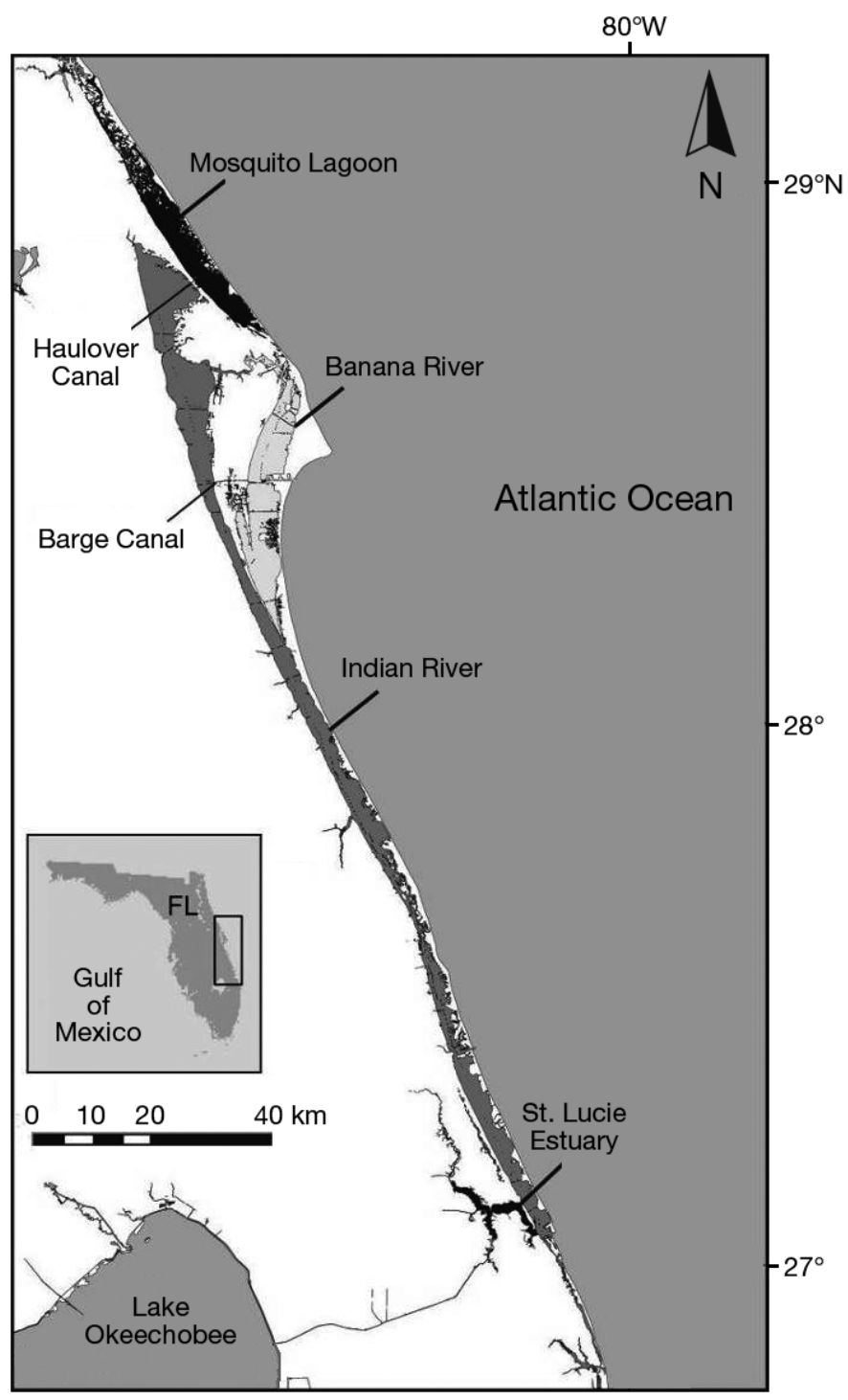

Fig. 1. Study site in the Indian River Lagoon, Florida (USA)

The CHS study site is situated in the central region of the state's coastal zone and consists of portions of the CHS Harbor estuary and the Stono River estuary (Fig. 2). Standardized stranding data have been accumulated for dolphins in the CHS area since 1992. From 1992 to 1996, bottlenose dolphins accounted for the majority $(73 \%)$ of marine mammal strandings in South Carolina, with CHS representing the highest number (McFee \& Hopkins-Murphy 2002). Although the cause of mortality for the majority of bottlenose dolphins stranded in South Carolina is unknown, during 1997 to 2003, human interaction accounted for $25 \%$ of dolphin strandings (McFee et al. 2006). Further analysis by McFee \& Lipscomb (2009) showed that $31 \%$ died from infectious diseases and $47 \%$ from non-infectious diseases. Bacterial infec- 


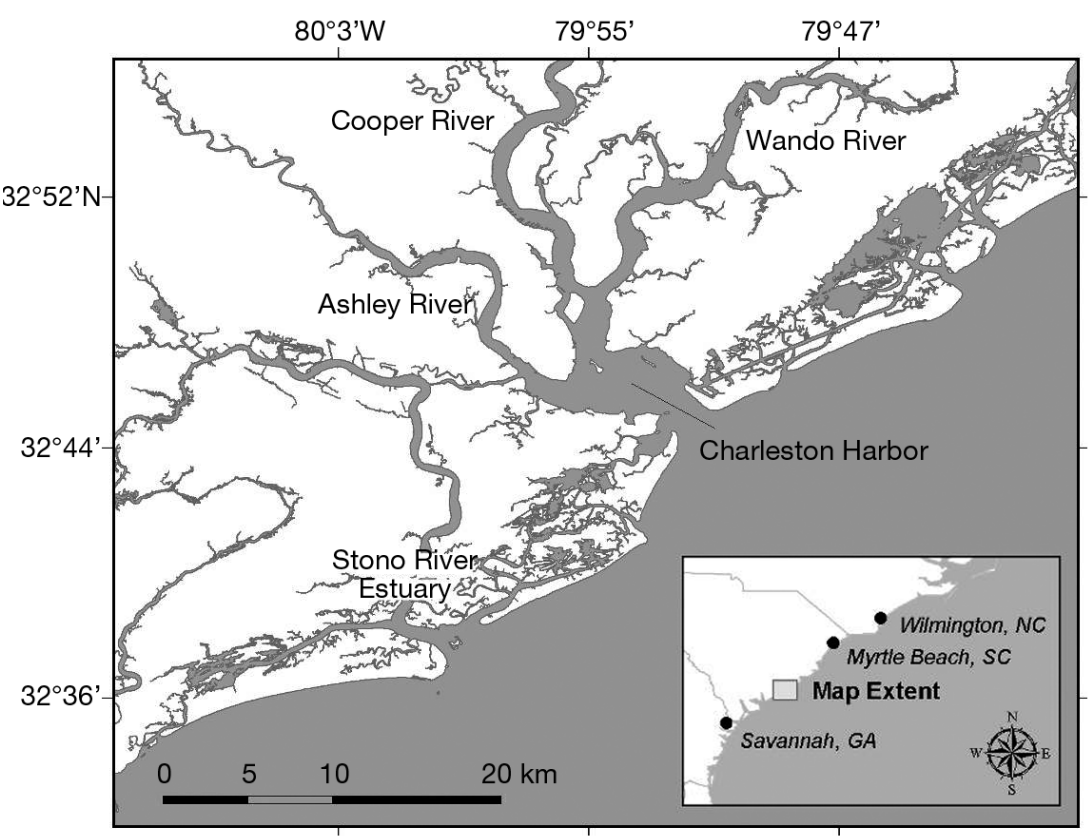

Fig. 2. Study site in Charleston, South Carolina (USA) presence of 2 distinct populations (genetic clusters) within the IRL (Richards et al. 2013). The information regarding genetic clusters became available in 2013. Therefore, with the exception of the 2015 HERA capture and release in the northern IRL, genetic data were not available for consideration.

\section{DOLPHIN HEALTH ASSESSMENTS}

Dolphins were captured, sampled, and safely released in the IRL during June of 2003-2007, 2010-2012, and 2015 and in the waters near CHS during August of 2003-2005 and 2013. Sampling strategy and standard operating procedures and techniques used for capture/release and sample collection are described in detail else- tions were the primary cause of infectious disease mortality, and emaciation was the leading cause of non-infectious mortality.

Photo-identification survey data indicate that both IRL and CHS dolphins display long-term residency patterns and site fidelity with an estimated population of 1000 dolphins for the IRL (Mazzoil et al. 2008) and ranging both seasonally and annually between 202 and 652 for the CHS estuarine dolphins (Speakman et al. 2010). IRL dolphins display site fidelity to relatively broad geographic areas, with most dolphins spending time in more than one IRL segment (Mazzoil et al. 2008). Because of their residency patterns, the populations of IRL and CHS dolphins are suitable for assessing risks associated with environmental factors. Differences in patterns of agriculture, urbanization, and concentrations of anthropogenic contaminants provide additional rationale for a comparative health assessment.

A recent study using microsatellite loci and mitochondrial DNA (mtDNA) control region sequences investigated patterns of genetic differentiation among the IRL and CHS dolphin populations. Additionally, the mtDNA was used to compare these populations with those from the Northwest Atlantic, Gulf of Mexico, and Caribbean. Results showed strong differentiation among inshore, alongshore, and offshore habitats. Both the mitochondrial and microsatellite data showed the CHS and IRL dolphin populations to be highly differentiated, and the microsatellite data for the first time revealed the where (Fair et al. 2006a). Briefly, study sites included all portions of the IRL. CHS capture sites included CHS Harbor, the Stono estuary, and mouths of the Ashley, Wando, and Cooper Rivers where they empty into Charleston Harbor (Fig. 2).

Sampling was conducted opportunistically as dolphins were sighted. Not all areas were sampled in each capture year, primarily for logistical and economic reasons and to comply with limitations on capture criteria and numbers specified in the NOAA Fisheries research permit. The numbers of dolphins evaluated in CHS was smaller than that for the IRL with a single capture year after 2005 (2013), which precludes a meaningful comparison within the CHS population. Most analyses considered the IRL as a single population and CHS as a second population. An exception in the IRL was our analyses of paracoccidioidomycosis ceti where clinically we suspected the disease prevalence was higher in the southern IRL and we performed multiple analyses to confirm this observation (Murdoch et al. 2008).

Health assessment evaluation included measurement of body weight and morphometrics, monitoring of vital signs (heart rate, respiration rate, cutaneous body temperature), a physical examination by an experienced marine mammal veterinarian, and the collection of a suite of biologic samples including blood (complete blood count, serum chemistry analytes, serum protein electrophoresis, and a suite of immunologic parameters); gastric fluid, feces, and nasal sinus exudate (cytologic evaluation and micro- 
biologic and antibiotic sensitivity testing); urine (standard urinalysis); and blubber and skin biopsies (contaminant and histopathologic analyses). Further details of additional parameters evaluated are provided in previous publications including reference values for hematologic parameters and thyroid hormones developed for both populations (Fair et al. 2006b, 2011, Goldstein et al. 2006, 2012, Bossart et al. 2008, 2011, 2014, 2015, Reif et al. 2008, 2009, Schaefer et al. 2009). Based on physical examination findings and the results of laboratory analyses, health status categories were determined by a panel of marine mammal veterinarians as previously described (Reif et al. 2008). Dolphins were classified as clinically healthy (no abnormalities noted), possibly diseased (1 or more clinical abnormalities noted requiring further diagnostics), and definitely diseased (1 or more clinical abnormalities noted which would require medical treatment if in a managed setting). Age was estimated by counting post-natal dentine layers in an extracted tooth (Hohn et al. 1989). When a tooth was unobtainable, age was estimated from total body length ranges established for these 2 dolphin populations (McFee et al. 2012). Adults were defined as dolphins 6 yr of age or older and subadults from 3.5 to $5.9 \mathrm{yr}$, as described previously (Bossart et al. 2008). Calves and pregnant dolphins in the third trimester were not included due to permit restrictions. All methods were approved under US National Marine Fisheries Service Scientific Research Permit Nos. 998-1678 and 14352 issued to G. Bossart and Florida Atlantic University IACUC protocol number A10-13. Additional details for specific health-related findings described below are found in previous publications as cited.

\section{HEALTH STATUS CLASSIFICATION AND DISEASE PREVALENCE}

Health assessments were made on 360 dolphins (246 IRL/114 CHS) representing 224 males (154 IRL/ $70 \mathrm{CHS}$ ) and 136 females (92 IRL/44 CHS). Age categories included adult males (113 IRL/51 CHS), subadult males (41 IRL/19 CHS), adult females (68 IRL/26 CHS), and subadult females (24 IRL/18 CHS). The health status classification and prevalence of disease results were divided into 2 periods: 2003-2007 and 2010-2015, in part to determine whether the prevalence of disease and specific disorders had changed, and in part to reflect the 2 separate 5 yr research permits.

Health assessment categories for all captures during the 5 yr period 2003-2007 are shown in Table 1. During this period, the prevalence of definite disease was higher in the IRL $(36 \%)$ than at CHS $\left(21 \% ; \chi^{2}-\right.$ test, $\mathrm{p}=0.03$ ).

Data for the 5 yr period 2003-2007 were analyzed by year of capture to evaluate temporal trends in disease prevalence (Table 2). The prevalence of definite disease among first captures increased at both sites between 2003 and 2005. At CHS, the prevalence of definite disease increased from $8.5 \%$ in 2003 to $33.3 \%$ in 2005, a 3.9-fold increase $(\mathrm{p}<0.01$ for the trend). In the IRL, the prevalence of definite disease increased from 28.6 to $50.0 \%$ between 2003 and 2005 but stabilized somewhat in 2006 and 2007. For the entire $5 \mathrm{yr}$ of observation, the prevalence of definite disease in the IRL was $32.5 \%$ on initial capture.

Two infectious diseases accounted for a substantial proportion of the observed morbidity between 2003 and 2007. Paracoccidioidomycosis ceti (PC; the re- 
cently proposed name of the disease caused by a novel uncultivated strain of Paracoccidioides brasiliensis and historically named lacaziosis/lobomycosis; Vilela et al. 2016) and orogenital papillomatosis (OP) accounted for two-thirds (67\%) of the diagnoses of definite disease among first captures. Eleven percent of dolphins captured in the IRL had PC, which was not identified among dolphins at the CHS site. OP was diagnosed in $12 \%$ from both capture sites. In addition, papillomas were detected in 10 of 28 repeat examinations, an incidence of $36 \%$.

The remaining dolphins with indicators of definite disease were classified on the basis of other clinicopathologic findings (Reif et al. 2008). The most common abnormalities were in the complete blood counts in which leukocytosis with absolute neutrophilia, lymphocytosis, and/or eosinophilia was the most consistent finding. Other abnormalities included regenerative anemia, low serum alkaline phosphatase, low serum iron, abnormal immunoglobulin profiles, or abnormalities in other serum analytes, particularly elevation in hepatic transaminases. Cytologic evidence of severe neutrophilic gastric inflammation was also found (Reif et al. 2008).

The prevalence of disease in the IRL and CHS dolphins from 2010 to 2015 is shown in Table 3. There was no significant difference between the prevalence of definite disease between the IRL and CHS for 2010 to 2015, although the data for CHS were based on a single capture year.

With respect to temporal patterns, the prevalence of definite disease in dolphins from both sites in 2010 to 2015 was not significantly different from the earlier reporting period ( 37 vs. $20 \%$ for CHS, 29 vs. $33 \%$ for the IRL; Table 3). The data suggest that morbidity in both populations remains elevated, but is relatively stable. Several of the infectious diseases responsible for much of the morbidity are endemic, e.g. OP in both populations and PC in the IRL (Bossart et al.

Table 3. Temporal trends in the prevalence of definite disease in bottlenose dolphins Tursiops truncatus by capture year and site, 2010-2015

\begin{tabular}{|c|c|c|c|c|c|c|}
\hline \multirow[t]{2}{*}{ Year } & \multicolumn{3}{|c|}{- Charleston -} & \multicolumn{3}{|c|}{ _Indian River Lagoon } \\
\hline & Cases & Number & $\begin{array}{c}\text { Prevalence } \\
(\%)\end{array}$ & Cases & Number & $\begin{array}{c}\text { Prevalence } \\
(\%)\end{array}$ \\
\hline 2010 & - & - & - & 8 & 26 & 30.8 \\
\hline 2011 & - & - & - & 6 & 27 & 22.2 \\
\hline 2012 & - & - & & 6 & 18 & 33.3 \\
\hline 2013 & 7 & 19 & 36.8 & - & - & - \\
\hline 2015 & - & - & - & 6 & 18 & 33.3 \\
\hline Total & 7 & 19 & 36.8 & 26 & 89 & 29.2 \\
\hline
\end{tabular}

2005, 2008, 2015, Reif et al. 2006). Interestingly, the results from assessments of health status from 2003 to 2015 did not show substantial differences between CHS and the IRL; much of the disease burden in both populations was due to infectious diseases.

The findings reported to date represent only the first phase of risk assessment employing standard methods of physical examination and clinicopathologic testing. An assessment of mortality is difficult due to the small number of HERA dolphins that died, were recovered, and were in a condition adequate to conduct a thorough post mortem examination. We suspect that many deceased dolphins are never recovered due to shark predation and other factors. A relatively high proportion of dolphins sampled had definite disease in both populations. This high prevalence may indicate that environmental stressors, albeit of differing origins, are impacting both populations.

\section{Cetacean morbillivirus (CeMV)}

Sera from IRL and CHS dolphins captured between 2003 and 2007 were tested for antibodies to CeMV. Positive CeMV titers were found only in IRL dolphins $(12 \%)$. Importantly, we found evidence for recurring cetacean CeMV infection in IRL HERA dolphins in the first post-CeMV epizootic (1987-1988) period, as well as evidence for chronic subclinical morbillivirus infection (Bossart et al. 2010). Two dolphins showed evidence of probable seroconversion to CeMV during the study, indicating that active infection had occurred between the sampling events. These dolphins were seronegative when sampled initially during 2006 and had positive titers to CeMV when resampled 1 yr later (Bossart et al. 2010). In addition, antibodies were detected against CeMV in IRL dolphins born after the 1987-1988 epizootic of morbillivirus, suggesting that CeMV infections were occurring in the absence of widespread mortality. Specifically, the prevalence of antibody to CeMV was $10 \%$ among dolphins born after 1988, providing evidence of subclinical infection during an inter-epidemic period (Bossart et al. 2010).

These data were consistent with the finding of CeMV infection in dolphins that stranded in the Gulf of Mexico from 1993 to 1994 (Lipscomb et al. 1996) and with serologic evidence of infection and recurrent epidemics in the Western Atlantic and Gulf of Mexico (Duignan et al. 
1996) through the mid-1990s. Interestingly, the first CeMV infections in stranded dolphins occurred in the Indian and Banana Rivers of the IRL in 1982 and preceded the massive die-off in 1987-1988 and further outbreaks in the Gulf of Mexico between 1992 and 1994. In retrospect, the 1982 increase in strandings of bottlenose dolphins in the IRL was associated with CeMV infection, based on the finding of antibodies in 5 of 6 dolphins sampled in that year and the absence of antibodies in 24 samples obtained from the same area in 1980 (Duignan et al. 1996).

A second, large epidemic of CeMV infection began along the eastern US seaboard in 2013 which was designated the 2013-2015 bottlenose dolphin UME in the Mid-Atlantic (NOAA Fisheries 2015). Increased numbers of stranded dolphins were observed along the coasts of New York, New Jersey, Delaware, Maryland, and Virginia, as the CeMV epidemic moved southward in a manner similar to that in 1987-1988. The total number of confirmed cases was 1374 through 30 June 2014 (Fauquier et al. 2015). Interestingly, between 2010 and 2012, 70 sera from IRL dolphins were tested for antibodies to CeMV and 6 sera were positive $(9 \%)$. The prevalence of seropositive animals was similar to that in our earlier analysis from 2003 to 2007 (10\%) (Bossart et al. 2010).

Cases of CeMV infection from the 2013-2015 UME were first diagnosed in stranded dolphins in the northern IRL in October 2013, suggesting transfer of CeMV from the coastal dolphin population to the relatively more isolated IRL dolphin population. CeMV cases continued to occur in Florida moving south until 2015 (NOAA Fisheries 2015). In June 2015, a HERA capture and release was undertaken in the same areas of the IRL where deaths from CeMV infection had occurred in 2014. Sera from 16 IRL dolphins were tested for antibodies against CeMV. All sera were negative. Additionally, no clinical abnormalities compatible with a morbillivirus infection were observed. All of the sera from CHS dolphins tested negative, thus this naïve population is vulnerable to CeMV outbreaks.

To further investigate CeMV infection in IRL dolphins, a suite of clinicoimmunopathologic variables was evaluated in dolphins seropositive for CeMV and seronegative healthy dolphins (Bossart et al. 2011). Several clinically significant differences were found. In CeMV seropositive dolphins, innate immunity appeared to be upregulated with significant increases in lysozyme concentration and marginally significant increases in monocytic phagocytosis. Adaptive immunity was also affected. Mitogen-induced T lymphocyte proliferation responses were significantly reduced in dolphins with positive CeMV antibody titers and marginally significant decreases were found for absolute numbers of CD4 lymphocytes. The findings suggested impairment of cell-mediated adaptive immunity, similar to the immunologic pattern reported with acute morbillivirus infection in other species (e.g. human measles, canine distemper) (Bossart et al. 2011). These data indicated that exposure to CeMV may result in subclinical dolphin infection in IRL dolphins producing immunologic changes that could influence overall health. The cell-mediated immunosuppressive effects associated with subclinical CeMV infection could make this dolphin population highly susceptible to opportunistic diseases. However, since the observed immunologic effects are not specific for CeMV infection, other undetected etiologic factors may also be responsible for the immunologic findings.

\section{Chlamydiaceae}

Chlamydiosis has not been reported in marine mammals. However, antibodies to Chlamydophila psittaci have been described in Steller sea lions Eumetopias jubatus (Burek et al. 2005) and in Hawaiian monk seals Monachus schauinslandi (Aguirre et al. 2007). In 2009, we described the first evidence of exposure to C. psittaci, or a closely related Chlamydiaceae in HERA dolphins (Schaefer et al. 2009). In this study, the seroprevalence of C. psittaci was 85 and $84 \%$ in dolphins from the IRL and CHS, respectively. Our follow-up study demonstrated that dolphins with a seropositive Chlamydiaceae antibody titer had several immunologic irregularities when compared to healthy dolphins (Bossart et al. 2014). Dolphins with positive Chlamydiaceae titers had significantly lower Con A-induced $\mathrm{T}$ lymphocyte proliferation, LPSinduced B lymphocyte proliferation, and granulocytic phagocytosis when compared to healthy dolphins. Dolphins with positive Chlamydiaceae titers also had significantly higher absolute numbers of CD21 mature B lymphocytes, NK activity, and lysozyme concentrations when compared to healthy dolphins. Additionally, dolphins with positive Chlamydiaceae titers had significantly lower total alpha globulin and alpha-2 globulin when compared with healthy dolphins. The results suggested partial up-regulation of innate immunity associated with Chlamydiaceae exposure. Adaptive immunity was also affected in dolphins with positive Chlamydiaceae antibody titers. The increased absolute numbers of CD 21 mature B cells in dolphins with positive Chlamydiaceae titers suggested a partial up-regulation of humoral immunity 
resulting from Chlamydiaceae exposure. However, mitogen-induced $\mathrm{B}$ and $\mathrm{T}$ lymphocyte proliferation responses were markedly decreased in dolphins with positive Chlamydiaceae titers, suggesting functional impairment of both humoral and cell-mediated immunity.

Dolphins with positive Chlamydiaceae titers also had significant increases in Erysipelothrix rhusiopathiae titers (Bossart et al. 2014). E. rhusiopathiae is a common marine microorganism that may cause disease in cetaceans (Bossart \& Eimstad 1988). Both E. rhusiopathiae and C. psittaci infections have been described in marine birds (Hubálek 2004). We speculated that the high seroprevalence of antibody to both Chlamydiaceae and E. rhusiopathiae in HERA dolphins might be caused by shedding of the agents among local bird reservoir populations which in turn may serve as potential sources of both chlamydial and E. rhusiopathiae infection (Bossart et al. 2014). The data suggest inapparent Chlamydiaceae infection impacts immunologic status which could make infected IRL and CHS dolphins susceptible to other diseases. Thus, both Chlamydiaceae and CeMV infections could impact the overall health of dolphins as described above.

\section{Arboviruses}

A summary of the prevalence of antibodies to a variety of other bacterial, protozoal, and viral pathogens from HERA dolphins has been published (Schaefer et al. 2009). Antibodies to several arthropod-borne viruses including West Nile virus and Venezuelan, western, and eastern equine encephalitis viruses were detected in IRL dolphins, representing the first reports of these pathogens in wild cetacean populations. The arboviruses examined in this study are commonly found in Florida, and the public health risk posed by these arboviruses is well documented (Day et al. 1996, Schaefer et al. 2009). Evidence of exposure to these pathogens in the dolphin population may indicate mosquito bite transmission to dolphins from infected bird reservoirs in the same geographical regions as human case activity (Schaefer et al. 2009).

\section{Paracoccidioidomycosis ceti}

Historically, lobomycosis (or lacaziosis) was considered a chronic fungal disease of the skin and subcutaneous tissues that occurred only in dolphins and humans under natural conditions. The presumed etiologic agent, Lacazia loboi, was described as a yeast-like organism found abundantly within lesions but not able to be cultured in vitro (Taborda et al. 1999, Schaefer et al. 2016). As a result, the development of diagnostic tools to help elucidate disease pathogenesis and epidemiology was impeded. In both species, the disease had a similar appearance of cutaneous keloidiform, white raised nodules that grew slowly over many years (Reif et al. 2006) (Fig. 3). Histologically, dolphin and human lesions consist of multifocal dermal granulomas with inflammatory infiltrates containing histiocytes, multinucleated giant cells, and large numbers of yeast-like cells, 6 to $12 \mu \mathrm{m}$ in diameter, arranged singly or in chains connected by tube-like bridges (Migaki et al. 1971, Bossart 1984). While historically the lesions and causative organisms appeared morphologically similar, initial gene sequencing of the organism from dolphins suggested it was more closely related to Paracoccidioides brasiliensis than to L. loboi of human origin (Rotstein et al. 2009). More recent molecular data from HERA dolphins and others supported these preliminary findings and show that a novel uncultivated strain of $P$. brasiliensis is the cause of dolphin lacaziosis/lobomycosis, which has now been renamed PC (Vilela et al. 2016). Previously, lobomycosis was often referred to as a zoonotic disease because it affected delphinids and humans. The new findings explain why the endemicity and natural history of PC in dolphins and lobomycosis in humans are strongly contrasted with very weak evidence of disease transmission directly to humans from dolphins (Reif et al. 2013).

In our initial analyses of data from HERA 2003 to 2004, we documented 9 cases of PC among the 75 IRL dolphins examined, a prevalence of $12 \%$ (Reif et

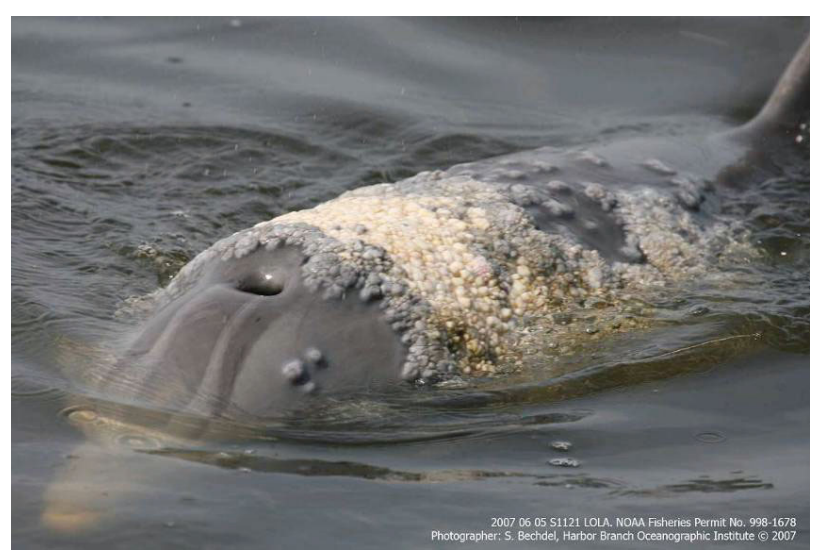

Fig. 3. Bottlenose dolphin Tursiops truncatus with paracoccidioidomycosis ceti from the Indian River Lagoon, FL 
al. 2006). This was the largest series of documented cases of PC reported to date and suggested that the disease may be emerging in the IRL. Further results from HERA showed that PC did not occur in the CHS environment, was rare in the northern IRL, and was endemic in the southern reaches of the IRL. Conditions in the IRL appear to favor PC, particularly in the southern segments of the estuary which are characterized by higher average water temperatures, runoff from agricultural watersheds, and freshwater intrusion (Woodward-Clyde Consultants 1994, Sime 2005). The prevalence of PC in IRL dolphins between 1996 and 2006 was also estimated from analysis of photo-identification images from 704 dolphins (Murdoch et al. 2008). The total prevalence was $7 \%$, and the spatial concentration of cases in the southern IRL was confirmed in this larger series. Presumptive cases were counted in the first year during which a lesion was identified to estimate incidence which did not increase over the course of the study period.

In an effort to characterize the pathogenesis of PC, we evaluated markers of immune status in HERA dolphins (Reif et al. 2009). Dolphins with PC had multiple abnormalities in hematologic and immunologic parameters when compared to normal dolphins. The absolute number of circulating lymphocytes and serum albumin concentration were reduced; segmented neutrophils, total serum globulins, alpha 1, total beta, total gamma globulins, and total protein were increased. Increases in globulins suggested that affected dolphins had an acute-phase inflammatory reaction or hypersensitivity response. Innate immunity parameters were relatively intact; phagocytosis and natural killer cell activity were not significantly different, but plasma lysozyme concentrations were elevated in dolphins with PC. Adaptive immunity was depressed in affected dolphins; statistically significant decreases were found in the absolute numbers of CD4+ helper T cells and CD19+ and CD21+ B cells. The ratios of CD2+ T cells to CD4+ cells and CD2+ to CD21+ cells were increased and the numbers of lymphocytes expressing MHC class II molecules were decreased in affected dolphins. Lymphocyte proliferation was reduced in response to stimulation with lipopolysaccharide and concanavalin A and antibody responses to common marine microorganisms, e.g. Erysipelothrix rhusiopathiae, were decreased. Taken together, the findings indicated that dolphins with PC exhibited significant immunologic impairment in adaptive immunity (Reif et al. 2009). The immune dysfunction associated with PC may reflect the host response to infection with this novel strain of $P$. brasiliensis. Alternatively, an acquired immunodeficiency may render dolphins susceptible to opportunistic $P$. brasiliensis infection as previously suspected (Bossart 1984). Specifically, an acquired immunodeficiency secondary to high concentrations of mercury is discussed elsewhere as a hypothesis for the pathogenesis of PC (Reif et al. 2017)

An alternative hypothesis is that infection with $P$. brasiliensis results in an altered immune system in which infection with the pathogen switches the host response from a Th1 to a Th2 response (Reif et al. 2009). Th1 cells, which produce interferon (IFN)- $\gamma_{\text {, }}$ interleukin (IL)-2, and tumor necrosis factor (TNF)- $\beta$, evoke cell-mediated immunity and phagocytedependent inflammation. Th2 cells, which produce IL-4, IL-5, IL-6, IL-9, IL-10, and IL-13, evoke IgE antibody responses and eosinophil accumulation, but inhibit several functions of phagocytic cells (phagocyte-independent inflammation). The lesions found in dolphins with PC are characterized by granulomatous inflammation containing numerous histiocytes and multinucleated giant cells and abundant numbers of yeast-like organisms (Bossart 1984, Bossart et al. 2003, 2015, Reif et al. 2006). Therefore, the inflammatory response to $P$. brasiliensis in dolphins appears to be ineffective in destroying the organism and preventing progression of the lesions. Similarly, the nodular skin lesions in human patients with lobomycosis are characterized by granuloma formation and infiltration of $\mathrm{CD}^{+} 8^{+}$histiocytes, CD4+ T cells, multinucleated giant cells, and to a lesser extent B cells (Vilani-Moreno et al. 2005). The cytokines observed in human lobomycosis are consistent with a Th2 response (Vilani Moreno et al. 2004). Thus, a similar mechanism could facilitate the growth of the fungus and prevent an effective immune response by switching to a Th2 pattern in PC.

\section{Orogenital papillomatosis}

We detected orogenital neoplasms in dolphins from the IRL and CHS during the initial phases of the HERA Project (Bossart et al. 2005). This was the first report of genital papillomas in free-ranging bottlenose dolphins from Atlantic coastal waters. Tumors were found primarily in adult dolphins of both sexes. Oral tumors occurred as single as well as multiple lesions located on the anterodorsal aspect of the tongue (Fig. 4). Genital lesions were found on the penis or external male genital mucosa or on the external female genital mucosa beneath the genital slit (Fig. 5). One dolphin had a genital 


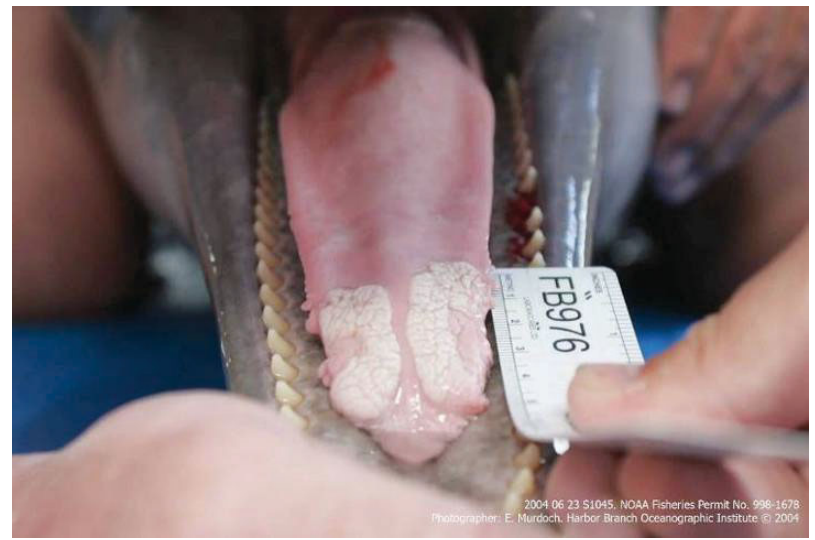

Fig. 4. Multiple lingual papillomas in an Indian River Lagoon bottlenose dolphin Tursiops truncatus

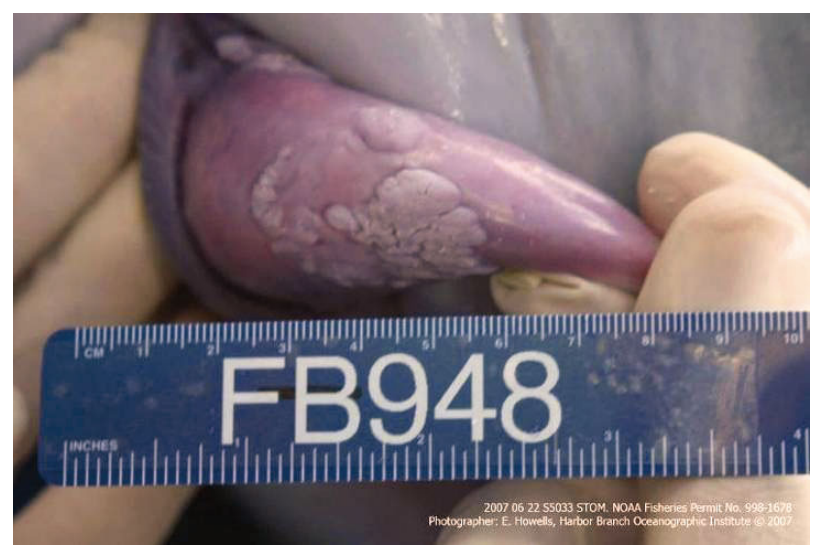

Fig. 5. Multiple genital papillomas on the penis of an Indian River Lagoon bottlenose dolphin Tursiops truncatus

sessile papilloma biopsied on the first examination that was not present on a re-examination 8 yr later. Microscopically, all oral and genital tumors were sessile papillomas.

Transmission electron microscopy revealed the presence of herpesvirus-like intranuclear particles and enveloped cytoplasmic virions in all papillomas (Bossart et al. 2005). In addition, a novel papillomavirus (PV) was isolated from a genital papilloma from a HERA dolphin and a complete nucleotide sequence of the virus designated as Tursiops truncatus papillomavirus type 2 (TtPV-2) was determined (Rehtanz et al. 2006). This virus represented the first identified North American cetacean PV. Polymerase chain reaction (PCR) analyses of genital papillomas revealed the presence of delphinid herpes virus-4 (DeHV-4) while serological screening using an antibody-based TtPV enzyme-linked immunosorbent assay (ELISA) demonstrated previous and/or current infection of the HV-positive dolphins with at least one TtPV type
(Rehtanz et al. 2009, 2012). The TtPV ELISA was also used to assess the extent of dolphin PV infection in the IRL and CHS (Rehtanz et al. 2010). Ninety percent of free-ranging dolphins sampled in the IRL and CHS were antibody positive. Higher ELISA reactivity was observed among males compared with females. Sexually immature dolphins appeared more likely to seroconvert with age, and the mean age of dolphins with tumors was 11 yr. A high PV antibody prevalence was not necessarily associated with tumor development as shown by serologic evidence of PV infection in $76 \%$ of IRL dolphins without genital tumors. The data indicate that PV infection in HERA bottlenose dolphins is common and readily transmitted horizontally between dolphins, most likely through sexual contact (Bossart et al. 2008, Rehtanz et al. 2010). The high level of sexual activity and its central role in play and social ordering of schools among cetaceans (Wells et al. 1987) may support the maintenance of viruses in those populations.

With respect to viral etiology of cancer, HERA research produced the first evidence of tumor-associated combined PV- and HV-infection and evaluated the controversial effects of viral co-infection in tumor pathogenesis, an important question of comparative human health significance (Rehtanz et al. 2012). Human PVs are the causative agents of cervical carcinoma, the most common cause of cancer mortality in women in developing countries and the second most frequent cancer in women worldwide. The role played by human HVs such as herpes simplex virus type 2 (HSV2) as co-factors in such cancers remains unclear, although HSV2 co-infection is regularly found in PV-induced cervical carcinomas. Eight species-specific viruses, 7 PVs, and 2 HVs have now been separately shown to be associated with genital tumors in Atlantic bottlenose dolphins: TtPV1-6, as well as HVs provisionally assigned the names DeHV4 and -5 (delphinid HVs) (Van Elk et al. 2009, Rehtanz et al. 2012). Therefore, it appears that the viral causality in tumor development is complex since 'hit and run' (i.e. no PV DNA detected after infection and neoplastic transformation) and other mechanisms have been described for members of both viral families (Rehtanz et al. 2012).

The prevalence of OP in both IRL and CHS populations increased dramatically during the first $3 \mathrm{yr}$ of the study, supporting the HERA laboratory data that an infectious agent(s) was driving disease emergence. HERA data now indicate that OP is an endemic disease in both populations. From 2010 to 2015, several dolphins were reexamined that had genital papillomas when first examined. In all cases, the 
lesions had resolved, indicating that in the wild, genital papillomatosis is probably a self-limiting disease. This is an important observation since it suggests that genital papillomas do not progress to carcinomas and therefore will not impact mortality. The regression of genital papillomas is consistent with what is commonly observed in domestic animals such as dogs and cows (Bossart et al. 2002, 2008). However, the biologic behavior of oral papillomas in free-ranging dolphins is less clear. While we have not observed mortality in free-ranging dolphins with oral papillomas, oral papillomas in dolphins under human care can undergo malignant transformation and progress to life-threatening metastatic cancer (Bossart et al. 2005, 2008, March et al. 2016). Additionally, malignant transformation of oral papillomas can occur after a prolonged time. Further monitoring is required to determine whether malignant progression of oral papillomas occurs in free-ranging dolphins. These studies show the importance of long-term follow up of disease conditions in marine mammal populations.

We also described the hematologic, biochemical, and immunologic findings in HERA dolphins with OP (Bossart et al. 2008). Compared to healthy dolphins without tumors, dolphins with OP demonstrated hypoferremia, hyperglobulinemia, and hyperalphaglobulinemia likely associated with an acute phase inflammatory response, upregulated innate immunity (i.e. significantly higher granulocytic and monocytic phagocytosis and superoxide respiratory burst), and upregulated humoral immunity (i.e. significantly higher B lymphocyte proliferation and antibody titers to the common marine microorganisms Escherichia coli, Erysipelothrix rhusiopathiae, Mycobacterium marinum, Vibrio cholerae, V. carchariae, V. vulnificus, and $V$. parahaemolyticus). Combined, these findings were possible responses to the tumors and/or the viruses associated with the tumors. Also, dolphins with OP may have enhanced innate and humoral adaptive immunity due to increased exposure to other pathogens (Bossart et al. 2008).

In a related study, we recently described mucocutaneous lesions identified in biopsies of HERA dolphins taken between 2003 and 2013 (Bossart et al. 2015). Pathologic diagnoses were OP $(40 \%), P C$ $(17 \%)$, tattoo skin disease (TSD) $(15 \%)$, nonspecific chronic to chronic-active dermatitis $(15 \%)$, and epidermal hyperplasia $(13 \%)$. This was the first longterm study documenting the various types, combined prevalence, and progression of mucocutaneous lesions in dolphins from the southeastern USA. Environmental and anthropogenic factors were consid- ered to play a role in the pathogenesis, diversity, and prevalence of many of these mucocutaneous lesions (Bossart et al. 2015)

\section{CONCLUSIONS}

Several studies of bottlenose dolphin populations in coastal US waters have evaluated various aspects of health. There are both similarities and differences in the methods used and in the research questions addressed by the investigators who conducted capture-release studies at various sites. In one instance, data from capture-release studies of 255 dolphins from 4 southeastern US coastal populations (IRL, CHS, Sarasota Bay, Florida, and Beaufort, North Carolina) were analyzed to establish reference intervals for hematologic and serum chemistry variables (Schwacke et al. 2009). The establishment of these reference values was made possible by the use of a single laboratory for all analyses.

The longest duration and most well-known of these studies is the Sarasota Dolphin Research Program, Sarasota Bay, Florida, which was originally designed as a long-term study of social structure and life history (Wells et al. 2004). Health and photo-identification data from Sarasota Bay dolphins have been used for multiple purposes, including descriptions of infectious diseases, microbial colonization, exposure to algal biotoxins, contaminant burdens, reproductive success and survival, and a variety of clinical conditions.

The US Navy Marine Mammal Program has maintained a population of bottlenose dolphins under managed care for over $50 \mathrm{yr}$. An evaluation of population mortality was conducted using data collected from 103 dolphins between 2004 and 2013 (VennWatson et al. 2015). The indicators analyzed included population age structures, annual survival, and crude mortality rates and median age at death. The analysis of these data as a retrospective cohort study was based on the long-term observation of individual animals over time, which differs from the crosssectional design used in HERA.

The oil spill caused by the explosion on the Deepwater Horizon in the Gulf of Mexico in 2010 prompted a series of studies to evaluate the health of bottlenose dolphins in the area. Health evaluations and pathologic examinations conducted on dolphins from Baratria Bay, Louisiana, demonstrated hypoadrenocorticism, lung disease, and an overall poor prognosis for survival, consistent with the effects of exposure to petroleum products (Schwacke 
et al. 2014). Longer-term follow up has shown decreased reproductive success and survival when compared to the data from Sarasota Bay dolphins (Lane et al. 2015).

Over the past $14 \mathrm{yr}$, the dolphin HERA study has generated a voluminous amount of traditional and novel health data which have provided important new information on individual and population dolphin health from 2 distinctly different estuarine regions of the southeastern USA. Interestingly, despite documented differences in observed diseases and concentrations of anthropogenic contaminants in the environment, the results from the health assessment did not show substantial differences between health status in CHS and IRL. The scientific publications generated from the HERA study have documented that both dolphin populations are impacted by newly characterized infectious and neoplastic disease, often associated with immunologic disturbances. Importantly, this in toto review of HERA data demonstrates the intricate and dynamic interactions that occur between infectious disease, anthropogenic contaminants, and/or immunologic responses. These interactions highlight the complexity of evaluating health in dolphins and may further complicate our understanding of the pathogenesis of disease and the detection of contributing factors in morbidity and mortality events, since the effect of any single factor may be confounded by other contributors. Furthermore, HERA research and other sustained wild dolphin health assessment programs have demonstrated that the bottlenose dolphin is a valuable sentinel species for understanding emerging or re-emerging diseases that may impact environmental and human health.

Acknowledgements. We thank the veterinarians, scientists, staff, and volunteers who participated in the HERA Project, with special thanks extended to Larry Hansen, George Biedenbach, Sarah Bechdel, Jennifer Bossart, Steve Burton, Chris Campbell, Dr. R.H. Defran, Matt Denny, Malcolm de Sieyes, Dr. Al Dove, Wendy Noke Durden, Alex Edlin, Robin Friday, Kerry Gladish, Dr. Juli Goldstein, Nicole Hatcher, Elisabeth Howells, Sharon Homer-Drummond, Dr. Lisa Hoopes, Dr. Brian Joseph, Dr. David Kilpatrick, Kenny Kroell, Marilyn Mazzoil, Wayne McFee, Dr. Craig Pelton, Dr. Lori Schwacke, Mark Simmons, Megan Stolen, Amanda Terry, Elizabeth Murdoch Titcomb, Dr. Forrest Townsend, Mark Trimm, Dr. Tim Tristan, Dr. Rene Varela, Harry Webb, Rob Yordi, and Eric Zolman. Finally, we gratefully acknowledge Stephen D. McCulloch for his dedication, tireless energy, and ongoing valuable contributions to marine mammal science. Support was provided by the Georgia Aquarium, the Florida Protect Wild Dolphins specialty license plate program, and the NOAA Fisheries Marine Mammal Health and Stranding Response Program.

\section{LITERATURE CITED}

Aguirre A, Keefe T, Reif J, Kashinsky L and others (2007) Infectious disease monitoring of the endangered Hawaiian monk seal. J Wildl Dis 43:229-241

Bossart GD (1984) A suspected acquired immunodeficiency in an Atlantic bottlenose dolphin with lobomycosis and chronic-active hepatitis. J Am Vet Med Assoc 185: 1413-1414

* Bossart GD (2011) Marine mammals as sentinel species for oceans and human health. Vet Pathol 48:676-690

* Bossart GD, Eimstad E (1988) Erysipelothrix vesicular glossitis in a killer whale (Orcinus orca). J Zoo Anim Med 19: 42-47

* Bossart GD, Ewing RY, Lowe M, Sweat M, Decker SJ, Walsh CJ, Ghim SJ (2002) Viral papillomatosis in Florida manatees (Trichechus manatus latirostris). Exp Mol Pathol 72: $37-48$

Bossart GD, Meisner R, Varela R, Mazzoil M, McCulloch S, Kilpatrick D, Friday R (2003) Pathologic findings in stranded Atlantic bottlenose dolphins (Tursiops truncatus) from the Indian River Lagoon, Florida. Fla Sci 66: 226-238

* Bossart GD, Ghim SJ, Rehtanz M, Goldstein J, Varela R, Ewing R, Fair P (2005) Orogenital neoplasia in Atlantic bottlenose dolphins (Tursiops truncatus). Aquat Mamm 31:473-480

Bossart GD, Goldstein JD, Murdoch EM, Fair PA, McCulloch SD (2006) Health assessment of bottlenose dolphins in the Indian River Lagoon, Florida and Charleston, South Carolina. Tech Rep 93. Harbor Branch Oceanographic Institute, Fort Pierce, FL

Bossart GD, Romano TA, Peden-Adams MM, Rice CD, Fair PA, Goldstein JD, Cammen K (2008) Hematological, biochemical and immunological findings in Atlantic bottlenose dolphins (Tursiops truncatus) with orogenital papillomas. Aquat Mamm 34:166-177

Bossart GD, Reif JS, Schaefer A, Goldstein J, Fair PA, Salikii JT (2010) Morbillivirus infection in free-ranging Atlantic bottlenose dolphins (Tursiops truncatus) from the southeastern United States: seroepidemiologic and pathologic evidence of subclinical infection. Vet Microbiol 143: 160-166

* Bossart GD, Romano TA, Peden-Adams MM, Schaefer A and others (2011) Clinicoimmunopathologic findings in Atlantic bottlenose dolphins Tursiops truncatus with positive cetacean morbillivirus antibody titers. Dis Aquat Org 97:103-112

Bossart GD, Romano TA, Peden-Adams M, Schaefer A and others (2014) Clinicoimmunopathologic findings in Atlantic bottlenose dolphins Tursiops truncatus with positive Chlamydiaceae antibody titers. Dis Aquat Org 108: 71-81

Bossart GD, Schaefer A, McCulloch S, Goldstein J, Fair PA, Reif JS (2015) Mucocutaneous lesions in free-ranging Atlantic bottlenose dolphins Tursiops truncatus from the southeastern USA. Dis Aquat Org 115:175-184

* Burek KA, Gulland FM, Sheffield G, Beckmen KB and others (2005) Infectious disease and the decline of Steller sea lions (Eumetopias jubatus) in Alaska, USA: insights from serologic data. J Wildl Dis 41:512-524

Way JF, Stark LM, Zhang J, Ramsey AM, Scott TW (1996) Antibodies to arthropod-borne encephalitis viruses in small mammals from southern Florida. J Wildl Dis 32: 431-436 
Duignan PJ, House C, Odell DK, Wells RS and others (1996) Morbillivirus infection in bottlenose dolphins: evidence for recurrent epizootics in the Western Atlantic and Gulf of Mexico. Mar Mamm Sci 12:499-515

Fair P, Adams J, Zolman E, McCulloch S and others (2006a) Protocols for conducting dolphin capture-release health assessment studies. Tech Memo NOS-NCCOS-49. US Department of Commerce, NOAA, Charleston, SC

Fair PA, Hulsey TC, Varela RA, Goldstein JD, Adams J, Zolman ES, Bossart GD (2006b) Hematology, serum chemistry and cytology findings from apparently healthy Atlantic bottlenose dolphins (Tursiops truncatus) inhabiting the estuarine waters of Charleston, South Carolina. Aquat Mamm 32:182-195

Fair PA, Montie E, Balthis L, Reif J, Bossart GD (2011) Influence of biological variables and geographic location on circulating concentrations of thyroid hormones in wild bottlenose dolphins (Tursiops truncatus). Gen Comp Endocrinol 174:184-194

Fauquier D, Goldstein T, Colegrove K, Rotstein DS and others (2015) Dolphin morbillivirus outbreak and the 2013-2014 mid-Atlantic bottlenose dolphin unusual mortality event. Wildlife Disease Association, Maroochydore, Queensland

Goldstein JD, Reese E, Reif JS, Varela RA and others (2006) Hematologic, biochemical, and cytologic findings from apparently healthy Atlantic bottlenose dolphins (Tursiops truncatus) inhabiting the Indian River Lagoon, Florida, USA. J Wildl Dis 42:447-454

Goldstein JD, Schaefer AS, McCulloch SD, Fair PA, Bossart GD, Reif JS (2012) Clinicopathologic findings from Atlantic bottlenose dolphins (Tursiops truncatus) with cytologic evidence of gastric inflammation. J Zoo Wildl Med 43: 730-738

Hersh SL, Odell DK, Asper ED (1990) Bottlenose dolphin mortality patterns in the Indian/Banana River system of Florida. In: Leatherwood S, Reeves RR (eds) The bottlenose dolphin. Academic Press, San Diego, CA, p 155-164

Hohn A, Scott M, Wells R, Sweeney JC, Irvine AB (1989) Growth layers in teeth from free-ranging, known-age bottlenose dolphins. Mar Mamm Sci 5:315-342

*Hubálek Z (2004) An annotated checklist of pathogenic microorganisms associated with migratory birds. J Wildl Dis 40:639-659

Lane SM, Smith CR, Mitchell J, Balmer BC and others (2015) Reproductive outcome and survival of common bottlenose dolphins sampled in Barataria Bay, Louisiana, USA, following the Deepwater Horizon oil spill. Proc Biol Sci 282:20151944

Lipscomb TP, Kennedy S, Moffett D, Krafft A and others (1996) Morbilliviral epizootic in bottlenose dolphins of the Gulf of Mexico. J Vet Diagn Invest 8:283-290

March DT, Blyde DJ, Bossart GD, Begg AP, Taylor DP, McClure V (2016) Piroxicam and doxycycline treatment for an oral squamous cell carcinoma in an inshore bottlenose dolphin (Tursiops aduncus). Aust Vet J 94:203-207

Marine Mammal Commission (2002) Annual Report to Congress, 2001. Marine Mammal Commission, Bethesda, MD

Mazzoil M, Reif JS, Youngbluth M, Murdoch ME and others (2008) Home ranges of bottlenose dolphins (Tursiops truncatus) in the Indian River Lagoon, Florida: environmental correlates and implications for management strategies. EcoHealth 5:278-288

McFee WE, Hopkins-Murphy SR (2002) Bottlenose dolphin (Tursiops truncatus) strandings in South Carolina, 19921996. Fish Bull 100:258-265
McFee WE, Lipscomb T (2009) Causes of mortality in bottlenose dolphins stranded in South Carolina from 19932006. J Wildl Dis 45:575-593

McFee WE, Hopkins-Murphy S, Schwacke L (2006) Trends in bottlenose dolphins (Tursiops truncatus) strandings in South Carolina, USA, 1997-2003: implications for the southern North Carolina and South Carolina management units. J Cetacean Res Manag 8:195-201

McFee WE, Adams JD, Fair PA, Bossart GD (2012) Age distribution and growth of two bottlenose dolphin (Tursiops truncatus) populations from capture-release studies in the southeastern United States. Aquat Mamm 38:17-30

*Migaki G, Valerio MG, Irvine B, Garner FM (1971) Lobo's disease in an Atlantic bottle-nosed dolphin. J Am Vet Med Assoc 159:578-582

Murdoch ME, Reif JS, Mazzoil M, McCulloch SD, Fair PA, Bossart GD (2008) Lobomycosis in bottlenose dolphins (Tursiops truncatus) from the Indian River Lagoon, Florida: estimation of prevalence, temporal trends and spatial distribution. EcoHealth 5:289-297

NOAA Fisheries (2015) 2013-2015 bottlenose dolphin unusual mortality event in the Mid-Atlantic. www.nmfs. noaa.gov/pr/health/mmume/midatldolphins2013.html

Rehtanz M, Ghim SJ, Rector A, Van Ranst M, Fair P, Bossart GD, Jenson AB (2006) Isolation and characterization of the first American bottlenose dolphin papillomavirus: Tursiops truncatus papillomavirus type 2. J Gen Virol 87: 3559-3565

Rehtanz M, Bossart GD, Doescher B, Rector A and others (2009) Bottlenose dolphin (Tursiops truncatus) papillomaviruses: vaccine antigen candidates and screening test development. Vet Microbiol 133:43-53

Rehtanz M, Ghim S, McFee W, Doescher B and others (2010) Papillomavirus antibody prevalence in free-ranging and captive bottlenose dolphins (Tursiops truncatus). J Wildl Dis 46:136-145

Rehtanz M, Bossart GD, Fair PA, Ghim S, Reif JS, Jenson AB (2012) Papillomaviruses and herpesviruses. Who is who in genital tumor development of free-ranging Atlantic bottlenose dolphins (Tursiops truncatus)? Vet Microbiol 160:297-304

Reif JS, Mazzoil M, McCulloch SD, Varela RA, Goldstein JD, Fair PA, Bossart GD (2006) Lobomycosis in Atlantic bottlenose dolphins (Tursiops truncatus) from the Indian River Lagoon, Florida. J Am Vet Med Assoc 228:104-108

Reif JS, Fair PA, Adams J, Joseph B and others (2008) Evaluation and comparison of the health status of Atlantic bottlenose dolphins from the Indian River Lagoon, Florida, and Charleston, South Carolina. J Am Vet Med Assoc 233:299-307

Keif JS, Peden-Adams MM, Romano TA, Rice CD, Fair PA, Bossart GD (2009) Immune dysfunction in Atlantic bottlenose dolphins (Tursiops truncatus) with lobomycosis. Med Mycol 47:125-135

* Reif JS, Schaefer AM, Bossart GD (2013) Lobomycosis: risk of zoonotic transmission from dolphins to humans. Vector Borne Zoonotic Dis 13:689-693

Reif JS, Schaefer AM, Bossart GD, Fair PA (2017) Health and Environmental Risk Assessment Project for bottlenose dolphins Tursiops truncatus from the southeastern USA. II. Environmental aspects. Dis Aquat Org 125:155-166

* Richards VP, Greig TW, Fair PA, McCulloch SD and others (2013) Patterns of population structure for inshore bottlenose dolphins along the eastern United States. J Hered 104:765-778 
Rotstein DS, Burdett LG, McLellan W, Schwacke L and others (2009) Lobomycosis in offshore bottlenose dolphins (Tursiops truncatus), North Carolina. Emerg Infect Dis 15:588-590

Schaefer AM, Reif JS, Goldstein JD, Ryan CN, Fair PA, Bossart GD (2009) Serological evidence of exposure to selected pathogens in free-ranging Atlantic bottlenose dolphins (Tursiops truncatus) from the Indian River Lagoon, Florida and Charleston, South Carolina. Aquat Mamm 35:163-170

Schaefer AM, Reif JS, Guzmán EA, Bossart GD and others (2016) Toward the identification, characterization and experimental culture of Lacazia loboi from Atlantic bottlenose dolphin (Tursiops truncatus). Med Mycol 54: 659-665

Schwacke LH, Hall AJ, Townsend FI, Wells RS and others (2009) Hematologic and serum biochemical reference intervals for free-ranging common bottlenose dolphins (Tursiops truncatus) and variation in the distributions of clinicopathologic values related to geographic sampling site. Am J Vet Res 70:973-985

Schwacke LH, Smith CR, Townsend F, Wells R and others (2014) Health of common bottlenose dolphins (Tursiops truncatus) in Barataria Bay, Louisiana, following the Deepwater Horizon oil spill. Environ Sci Technol 48: 93-103

Sime P (2005) St. Lucie Estuary and Indian River Lagoon conceptual ecological model. Wetlands 25:898-907

Speakman T, Lane SM, Schwacke LH, Fair PA, Zolman ES (2010) Mark-recapture estimates of seasonal abundance and survivorship for bottlenose dolphins (Tursiops truncatus) near Charleston, South Carolina. J Cetacean Res Manag 11:53-62

Stolen MK (1998) Age, growth, and mortality of bottlenose dolphins from the east coast of Florida. MSc thesis, University of Central Florida, Orlando, FL

Stolen MK, Durden WN, Odell DK (2007) Historical synthesis of bottlenose dolphin (Tursiops truncatus) stranding

Editorial responsibility: Steven Raverty, Abbotsford, British Columbia, Canada data in the Indian River Lagoon system, Florida, from 1977-2005. Fla Sci 70:45-54

Taborda PR, Taborda VA, McGinnis MR (1999) Lacazia loboi gen. nov., comb. nov., the etiologic agent of lobomycosis. J Clin Microbiol 37:2031-2033

Van Elk CE, Van de Bilt M, de Jong A, Osterhaus A, Kuiken $\mathrm{T}$ (2009) Genital herpesvirus in bottlenose dolphins (Tursiops truncatus): cultivation, epidemiology and pathology. J Wildl Dis 45:895-906

*Venn-Watson S, Jensen E, Smith CR, Xitco M, Ridgway SH (2015) Evaluation of annual survival and mortality rates and longevity of bottlenose dolphins (Tursiops truncatus) at the United States Navy Marine Mammal Program from 2004 through 2013. J Am Vet Med Assoc 246:893-898

*Vilani-Moreno FR, Lauris JR, Opromolla DV (2004) Cytokine quantification in the supernatant of mononuclear cell cultures and in blood serum from patients with Jorge Lobo's disease. Mycopathologia 158:17-24

*Vilani-Moreno FR, Belone AF, Soares CT, Opromolla DV (2005) Immunohistochemical characterization of the cellular infiltrate in Jorge Lobo's disease. Rev Iberoam Micol 22:44-49

* Vilela R, Bossart GD, St. Leger JA, Dalton LM and others (2016) Cutaneous granulomas in dolphins caused by novel uncultivated Paracoccidioides brasiliensis. Emerg Infect Dis 22:2063-2069

Wells RS, Scott MD, Irvine AB (1987) The social structure of free-ranging bottlenose dolphins. In: Genoways $\mathrm{HH}$ (ed) Current mammalogy. Plenum Press, New York, NY, p 246-254

*Wells RS, Rhinehardt HL, Hansen LJ, Sweeney JC and others (2004) Bottlenose dolphins as marine ecosystem sentinels: developing a health monitoring system. EcoHealth 1:246-254

Woodward-Clyde Consultants (1994) Indian River Lagoon Natural Estuary Program. Project Number: 92F274C. Prepared for the Indian River Lagoon National Estuary Program, Melbourne, FL

Submitted: January 16, 2017; Accepted: May 18, 2017 Proofs received from author(s): June 30, 2017 